Vlasov description of the collision between two slabs

This content has been downloaded from IOPscience. Please scroll down to see the full text.

1988 J. Phys. G: Nucl. Phys. 14205

(http://iopscience.iop.org/0305-4616/14/2/012)

View the table of contents for this issue, or go to the journal homepage for more

Download details:

IP Address: 193.136.215.65

This content was downloaded on 17/04/2017 at 16:39

Please note that terms and conditions apply.

You may also be interested in:

Variational formulation of the Vlasov equation

$\mathrm{J}$ da Providencia and C Fiolhais

Microscopic and Macroscopic Aspects of Nuclear Dynamics in Mean-Field Approximation. (I. Formalism)

V M Kolomietz and Henry H K Tang

Dynamics of nuclear integral characteristics

E B Balbutsev and I N Mikhailov

Surface modes in metal clusters and cavities

João da Providência Jr

Nuclear fluid dynamics of hot nuclei

JP da Providencia

Damping of small-amplitude nuclear collective motion

J Wambach

Dissipative features of the Vlasov equation in a simple model

P L'Eplattenier, E Suraud and P G Reinhard

Damping of the Giant Resonances in a Fluid-Dynamical Model

J. da Providência

Kinetic Theory for Evolution of CDM Halos

Chung-Pei Ma and Edmund Bertschinger 


\title{
Vlasov description of the collision between two slabs
}

\author{
$\mathrm{J}$ da Providência and $\mathrm{C}$ Fiolhais \\ Departamento de Física da Universidade de Coimbra, P-3000 Coimbra, Portugal
}

Received 7 May 1987

\begin{abstract}
A simple analytical description of the adiabatic collision between two slabs of nuclear matter is presented, as an illustration of a recent variational formulation of the Vlasov equation.
\end{abstract}

It is the purpose of this short paper to present a description of adiabatic largeamplitude collective motion of a nuclear system, within the framework of the Vlasov equation. We consider the collision along the $z$ axis of two slabs of nuclear matter, since this system has been treated extensively in TDHF theory, which is the quantal background of the Vlasov equation [1].

The Vlasov equation may be derived from an action principle $[2,3]$. This is the starting point for a variational solution in which the relevant degrees of freedom are taken into account by a hopefully suitable parametrisation of the trial distribution functions. We choose as variational fields the velocity potential $\phi(z, t)$, the density fluctuation around equilibrium $\rho_{1}(z, t)$, such that $\rho_{1}=\rho-\rho_{0}$, where $\rho$ is the actual density and $\rho_{0}$ the equilibrium one, and the displacement of the nuclear surface along the normal direction $\delta R=\delta \boldsymbol{R} \cdot \hat{k}$. The motion is irrotational, in view of the onedimensional geometry of the problem. We consider that the motion is slow enough that anisotropic deformations of the Fermi sphere may be disregarded. It is possible to improve this approximation by including further variational fields [4].

An equilibrium distribution function, which is the solution of the Thomas-Fermi problem, reads as

$$
f_{0}(z, p)=\theta\left(\lambda-p^{2} / 2 m-U_{0}(z)\right)
$$

where $\lambda$ is the Fermi energy and $U_{0}(z)=U_{0}\left(\rho_{0}(z)\right)$ the self-consistent mean-field potential, which is determined by the Hamiltonian of the system.

The following distribution function describes a system with a static deformation:

$$
f_{\mathrm{st}}(z, \boldsymbol{p}, t)=\theta\left(\lambda-\boldsymbol{p}^{2} / 2 m-U_{0}(z)-W(z, t)\right)
$$

where the field $W(z, t)$ is responsible for the density fluctuations and for the displacement of the nuclear surface. Considering $W(z, t)$ to be small, it is possible to relate it directly to $\rho_{1}: W \propto \rho_{1}$. We remark that although the amplitude of the surface motion may be large, it is assumed that the motion is slow enough that at each instant 
of time the static distribution function does not differ very much from an equilibrium solution.

The dynamically deformed distribution function reads as

$$
f(z, \boldsymbol{p}, t)=f_{\mathrm{st}}+\left\{\phi, f_{\mathrm{st}}\right\}+\frac{1}{2}\left\{\phi,\left\{\phi, f_{\mathrm{st}}\right\}\right\} .
$$

Let us consider for the system a Hamiltonian $H$ containing two- and three-body zero-range forces, which provides correct values for the Fermi momentum and the binding energy of nuclear matter [4]. In view of the locality of the interactions, there is no surface tension.

The lagrangian of the system is then

$$
L=\int\left(\rho_{1} \dot{\phi}-\frac{1}{2 m} \rho_{0} \nabla \phi \cdot \nabla \phi-\frac{m}{2} \frac{c^{2}}{\rho_{0}} \rho_{1}^{2}\right) \mathrm{d}^{3} r+\int \mathrm{d} S \rho_{0} \dot{\phi} \delta R
$$

where the second term is the collective kinetic energy, the third one the potential energy, the parameter $c$ being the velocity of propagation of $H$-sound disturbances (it is obtained from $H$ by evaluating the second functional derivative of the energy with respect to the density), and the last one accounts for the boundary motion. The lagrangian (4) is harmonic in both variational fields, in contrast with the lagrangian which leads to Bloch's equations [5]. The latter is quadratic in the velocity but its potential energy corresponds to the well known Thomas-Fermi (or extended Thomas-Fermi) functional between energy and static density. In both cases an adiabatic hypothesis is assumed, although in our case this is more drastic, preventing any anharmonic terms in the potential energy.

The variation of the lagrangian (4) leads to the following equations of motion

$$
\phi-\frac{m c^{2}}{\rho_{0}} \rho_{1}=0 \quad \dot{\rho}_{1}-\frac{\rho_{0}}{m} \nabla^{2} \phi=0
$$

and to the boundary conditions

$$
-\left.\frac{\partial \phi}{\partial z}\right|_{s}-m \delta \dot{R}=\left.0 \quad \dot{\phi}\right|_{s}=0
$$

The first of equations (5) is an Euler equation and the second a continuity equation. Combined, they yield a wave equation for the fields $\phi(z, t)$ and $\rho_{1}(z, t), c$ being the velocity of the wave. The first boundary condition assures that the surface follows the motion of the quasiparticles in the interior of the slab, while the second does not allow the surface to acquire an acceleration.

As an example of the utility of this dynamical scheme, we consider the motion against each other of two parallel slabs of different thicknesses. We begin with the case of a single slab in uniform motion along the $z$ axis and take as initial conditions for the two fields

$$
\phi(z, 0)=-\alpha z \quad \rho_{1}(z, 0)=0 .
$$


Since $\delta \dot{R}=-(1 / m) \partial \phi / \partial z=\alpha / m$, it follows from equations (5) and (6) that the slab is actually moving with velocity $\alpha / m$ along the $z$ direction. At time $t$, the velocity potential is

$$
\phi(z, t)=-\alpha\left(z-\frac{\alpha}{m} t\right)
$$

so that the distribution function is

$$
f(z, p, t)=\theta\left(\lambda-\frac{1}{2 m}(p-\alpha \hat{k})^{2}-U_{0}\left(z-\frac{\alpha}{m} t\right)\right) .
$$

Now let $a$ and $b$ be the thicknesses of two different slabs, and let $\varepsilon b$ and $-\varepsilon a$ be their respective velocities in the frame of the centre of mass ( $\varepsilon$ being a parameter small enough to make the collective velocity small compared with the sound velocity in the medium). At the instant $t=0$, when the two slabs touch each other, the velocity field is

$$
\begin{array}{ll}
\phi(z, 0)=-\varepsilon b(z+a) & z \in[-a, 0] \\
\phi(z, 0)=\varepsilon a(z-b) & z \in[0, b] .
\end{array}
$$

The density fluctuation and the surface displacement vanish at $t=0$ :

$$
\rho_{1}(z, 0)=0 \quad \delta R(t=0)=0 .
$$

In order to solve equations (5) and (6) with boundary conditions (10) and (11) we adopt the standard procedure of considering the normal modes of the fields $\phi(z, t)$ and $\rho_{1}(z, t)$ in the box $[-a, b]$. These eigenmode solutions are respectively

$$
\phi_{n}(z, t)=\sin \left[k_{n}(z+a)\right] \cos \left[c k_{n}(t-\varphi)\right]
$$

$\rho_{1, n}(z, t)=-\frac{\rho_{0}}{m} \frac{k_{n}}{c} \sin \left[k_{n}(z+a)\right] \sin \left[c k_{n}(t-\varphi)\right] \quad n=1,2,3, \ldots$

with the wavenumber

$$
k_{n}=n \pi /(a+b)
$$

Note that the modes $\phi_{n}$ are time-even, while the functions $\rho_{1, n}$ are time-odd. The initial phase $\varphi$ must vanish in view of the initial condition (11) fulfilled by the field $\rho_{1}$. 


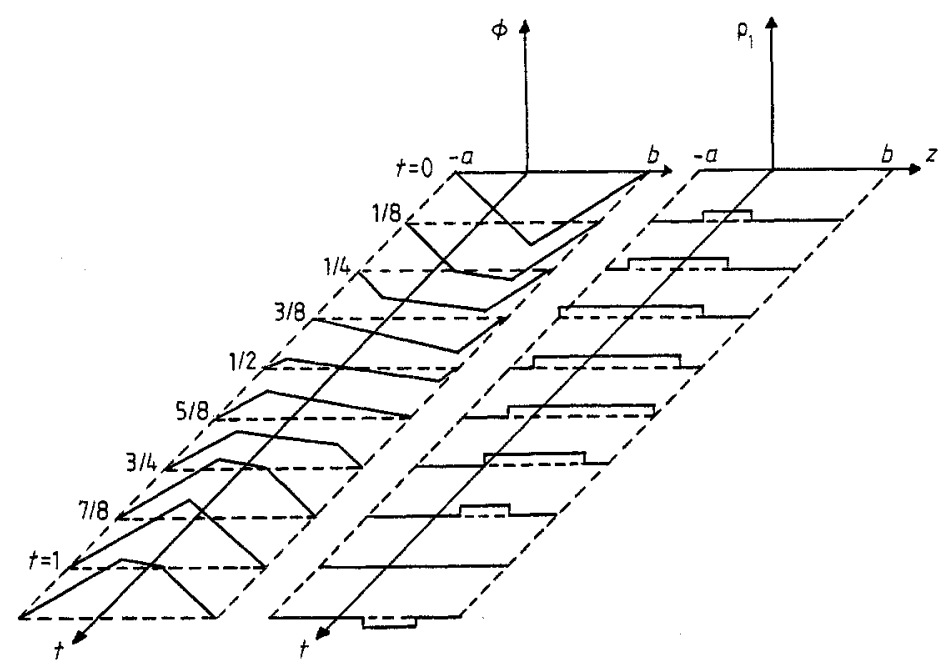

Figure 1. Time evolution of the velocity potential $\phi$ and the density fluctuation $\rho_{\text {a }}$ a function of $z$. The time unit is $(a+b) / c$.

The required solution for $\phi(z, t)$ may be expressed in terms of the normal modes or alternatively as a sum of one forward and one backward travelling function:

$$
\begin{aligned}
\phi(z, t) & =\sum_{n} A_{n} \sin \left[k_{n}(z+a)\right] \cos \left(c k_{n} t\right) \\
& =\frac{1}{2} \sum_{n} A_{n}\left\{\sin \left[k_{n}(z+a+c t)\right]+\sin \left[k_{n}(z+a-c t)\right]\right\} \\
& =\frac{1}{2}[\phi(z+c t)+\phi(z-c t)]
\end{aligned}
$$

where $\phi(z)=\phi(z, 0)$ may be continued for $z \in[-2 a, 2 b]$ :

$$
\begin{aligned}
& \phi(z)=-\varepsilon b(z+a) \quad z \in[-2 a, 0] \\
& \phi(z)=\varepsilon a(z-b) \quad z \in[0,2 b] .
\end{aligned}
$$

On the other hand, the density fluctuation may be written as

$$
\begin{aligned}
\rho_{1 . n}(z, t) & =-\frac{\rho_{0}}{m c} \sum_{n} A_{n} k_{n} \sin \left[k_{n}(z+a)\right] \sin \left(c k_{n} t\right) \\
& =\frac{\rho_{0}}{2 m c} \sum_{n} A_{n} k_{n}\left\{\cos \left[k_{n}(z+a+c t)\right]-\cos \left[k_{n}(z+a-c t)\right]\right\} \\
& =\frac{\rho_{0}}{2 m c^{2}} \frac{\partial}{\partial t}[\phi(z+c t)+\phi(z-c t)] .
\end{aligned}
$$

Figure 1 shows a sequence of snapshots at different times for the velocity potential and the density disturbance. In the region where the two slabs touch, a positive 
disturbance of the density appears, which first propagates towards the nearest boundary (reached at time $a / c$ ), then moves towards the other extreme and finally disappears at the position $b-a$. This front shock arises also in the much more involved TDHF calculations. At the time $t=(a+b) / c$, the velocity potential is

$$
\begin{array}{ll}
\phi\left(z, \frac{a+b}{c}\right)=\varepsilon a(z+a) & z \in[-a, b-a] \\
\phi\left(z, \frac{a+b}{c}\right)=-\varepsilon b(z-b) & z \in[b-a, b]
\end{array}
$$

and the density fluctuation vanishes as initially. The two surfaces are now moving outwards. According to our variational solution, the system becomes compound, with a negative disturbance arising at $z=b-a$, growing until the nearest boundary is reached and finally vanishing at $z=0$, when the initial conditions recur. The combined system remains oscillating back and forth.

The Vlasov description presented here predicts the fusion of the slabs after collision. It should be remarked, however, that in the absence of surface tension no energy is necessary to create two new surfaces along the line $z=b-a$. Therefore, our treatment is not inconsistent with the separation of the compound slab at $t=(a+b) / c$ into two pieces defined by the instantaneous pattern of the velocity field. The equations of motion assure that the subsequent motion of the two slabs is in opposite directions. Let us then examine how less restricted theories, such as TDHF and complete Vlasov, deal with the permanence of the compound slab.

It is known from TDHF numerical studies [1] of systems with the same geometry that for small kinetic energies per particle $(E / A \leqslant 1 \mathrm{MeV}$, in the $\mathrm{CM}$ frame), corresponding to the adiabatic hypothesis assumed here, the slabs actually fuse and that for high kinetic energies $(E / A \geqslant 4 \mathrm{MeV})$ the two slabs become transparent to each other. There is an intermediate region with a mixed behaviour, as a result of delicate shell effects. The numerical solution of the Vlasov equation for the same problem yields a result that agrees surprising well with TDHF [6]. On the other hand, Holzwarth [7] has solved Bloch's equations (sometimes also called time-dependent Thomas-Fermi equations) for the collision of two equal slabs, using an extended Thomas-Fermi energy functional. His results look qualitatively similar to TDHF or Vlasov ones. We should conclude that a modification of our harmonic potential energy is essential to correctly account for the break-up of the compound system. Nevertheless, the TDHF results corresponding to very high energies should be taken with care, since particle collisions, not included in the mean field, are important in that regime.

We have described analytically the fusion at low energies of two colliding slabs, using a variational formulation of the Vlasov equation. Numerical instabilities, which are characteristic of more sophisticated approaches, are absent in the present approach. The kinetic energy was shown to be transformed in undamped wave motion. With respect to one-body damping, we point out that a richer parametrisation of the distribution function may be implemented, leading to more than one velocity of sound. The recurrence of a given situation then becomes more unlikely. A short recurrence time is typical of models, such as ours, with a restricted geometry and with restricted dynamics, allowing only for one kind of sound. 


\section{Acknowledgment}

This work has been supported by the Instituto Nacional de Investigação Científica, Lisbon, and by the C Gulbenkian Foundation.

\section{References}

[1] Bonche P, Koonin S and Negele J 1976 Phys. Rev. C 131226

[2] da Providência J, Brito L and da Providência C 1985 Nuovo Cimento A 87248

[3] da Providência J and Fiolhais C 1987 J. Phys. A: Math. Gen. 203877

[4] Brito L and Providência C 1985 Phys. Rev. C 322049

[5] Bloch F 1933 Z. Phys. 81363

[6] Wong C-Y 1982 Phys. Rev. C 251460

[7] Holzwarth G 1977 Phys. Lett. 66B 29 